\title{
Blur - Map Estimation and Deblurring of Degraded Image by Non - Convex Regularization Approach
}

\author{
Latha $\mathrm{H} \mathrm{N}^{1,2} \mid$ Rajiv R Sahay ${ }^{3}$ \\ ${ }^{1}$ Department of Computer Science, IIT, KGP, West Bengal, India \\ ${ }^{2}$ Department of E \& C, BMS College of Engineering , Bangalore, Karnataka, India \\ ${ }^{3}$ Department of E \& E, IIT, KGP, West Bengal, India
}

\section{To Cite this Article}

Latha H N and Rajiv R Sahay, "Blur - Map Estimation and Deblurring of Degraded Image by Non - Convex Regularization Approach”, International Journal for Modern Trends in Science and Technology, 6(8S): 134-139, 2020.

\section{Article Info}

Received on 16-July-2020, Revised on 15-August-2020, Accepted on 25-August-2020, Published on 28-August-2020.

\section{ABSTRACT}

We in this work proposed a new algorithm for blur-map estimation of space variant degraded image using a graduated non convex discontinuity adaptive Markov random field (DAMRF) frame work. First we estimated to find the blur-map and then restore the blurred image in. We use a GNC optimization based framework, wherein we assume the latent image and blur map as DAMRF prior. We show that the estimated blur-map is utilized for image restoration application such as image in-painting, depth estimation, image denoising, super resolution, haze removal etc. Robust estimation of the non-uniform blur-map of a natural scene is obtained produces better qualitative and quantitative results compared to the few state-of-the method

KEYWORDS: Graduated Non Convex 1, Space-variant Blur 2, Markov Random Field 3, Discontinuity Adaptive 4, Non-Convexity 5.

\section{INTRODUCTION}

Non-blind image deconvolution is a well-known frequently addressed ill-posed issue in image restoration. Accurate blur-map estimation is required to reduce the commonly occurring ringing artifacts during deblurring of degraded image due to optical image-based systems. In many recent papers, authors proposed differentwork [1]- [6] to solve such inverse problem.

The addressed inverse image restoration problem solved are classified as Bay'soptimization framework, Max-a-posterior, Dictionary learning using sparse code, KSVD algorithms, Foreground and background segmentation using Alpha matings, selection of strong edge, by using blurred and noisy multi-frame, DCNN models.

Inverse blind deblurring by single degraded image has strained more authors during previous last decades to solve such issues. Image capturing devices such as portable hand-held mobile cameras, image sensor equipment'sare generallysuffers from defocusing due to camera lens, which result in blurring of naturals scent images when capturing. Blurring basically happens due to camera motion or object motion. Blurring is classified by the rotation or translation motion between image capturing device and target scene in lens during camera lens shutter open exposure time

Blur operations are classified as space-variant or pace-invariant. Uniform blur through-out the image is referred as space-in-variant. In case of space-variant blurring each and every pixel could have different amount of blur details. The blur processing operation is modeled as a convolution functions as given by 


\section{PROBLEM DEFINITION AND PROPOSED}

$$
\mathrm{y}=\mathrm{H} \otimes \mathrm{x}+\eta
$$

Where, yrepresent blurry input, $\mathrm{x}$ is latent image, $\mathrm{H}$ is blur kernel and $\eta$ is the AWGN [11] noise, respectively, and $\otimes$ denotes the convolution symbol. Latent image $\mathrm{x}$ and blur kernel $\mathrm{H}$ ofthis equation is generally solved by suitable optimization based frame work.

\section{PREVIOUS WORK AND LITERATURE}

Authors [7]- [10] proposed a MAP framework using total variation Bayes models to deblur the motion degraded image using inverse transform algorithms for a fast motion deblurring issues. Utilized by [11]-[15] andifferent L1 or L2 normalization workswith convex and non-convex regularization to solve inverse issues.

In [16]-[23] represented a non-statics dynamic approach using variable saliency models for detection of strong edges and minute details at low frequency components of the images

The addition of priors in regularization framework is w.r.t. shape and structure details of the PSF proposed by authors [24]-[30], showed removal of defocusing and motion blurring of partially blurred in a multiform/single image presented a maximal similarity estimation method that reduce the window effect. Our objective in this is to estimate the blur-map initially and then the given blurred image is restored. Microscopic cameras can be used for obtaining the defocused images. We use blurred images and the focused image is estimated using JNB [4] as well as Gauss Markov Random field algorithm. Then, from the focused image, the blur map is estimated and subsequently the depth of the image.

Our main contribution to this work is a robust novel method to detect and estimate blur using just noticeable blur approach. We use this method as a base to retrieve the focused image. Using the alternating minimization optimization framework all-in-focus image is reconstructed. From the blurred observation we can also estimate depth-map, for image in-painting problems. We use gradient descent with discontinuity adaptive Markov random field [12] prior along with graduated non-convexity algorithm to estimate blur-map, thus preserving fine details and solving the optimization problem.
Initially the problem of blur-map estimate is decomposed into three steps. Firstly the rough blur-map is generated by JNB. Secondly using gradient based techniques image is deblurred. Finally by GNC-DAMRF optimization framework in alternating method is implemented to get latent image.

\section{A. Image deblurring model}

In this case, blurring is modeled for unknown image $\mathrm{x}$ as :

$$
y=H x+\eta
$$

The problem of estimation can be formulated as the minimization of the

$$
E=\frac{1}{2}\|y-H x\|_{2}^{2}+\lambda R(d)
$$

energy function given by

$$
\frac{1}{2}\|y-H x\|_{2}^{2}=\frac{1}{2}\left(y^{T} y-2 y^{T} H x+x^{T} H^{T} H x\right)
$$

(4)

The gradient descent update is

$$
\begin{array}{r}
x_{\text {new }}=x_{\text {old }}-\alpha \frac{\partial E}{\partial x} \\
x_{\text {old }}=x_{\text {new }}
\end{array}
$$

where $a$ is the learning rate or the step size. Image deblurring algorithm is as given below

$$
E=\frac{1}{2}\|y-H x\|_{2}^{2}+\lambda R(d)
$$




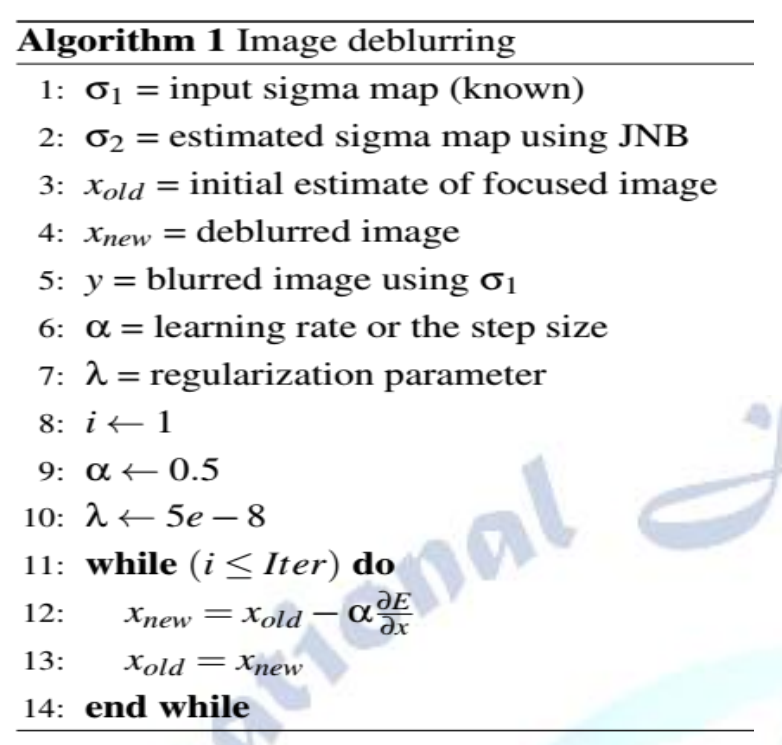

\section{B. Discontinuity adaptive prior (DAMRF)}

The priors of discontinuity adaptive MRF (DAMRF) is obtained by the following regularization function.

$$
R(d)=\gamma-\gamma e^{-\eta^{2} / \gamma}
$$

$\mathrm{R}(\mathrm{d})$ is a convex function in the range $(\sqrt{-\gamma / 2}, \sqrt{\gamma / 2})$.

$$
\begin{gathered}
\text { So, } E=\frac{1}{2}\left\|y-X h_{k_{p}}\right\|_{2}^{2}+\lambda R(d) \\
\sum_{c \in C} V_{c}(x)=\sum_{i} \sum_{j} 4 \gamma-\gamma e^{-[x(i, j)-x(i, j-1)]^{2} / \gamma} \\
-\gamma e^{-[x(i, j)-x(i, j+1)]^{2} / \gamma} \\
-\gamma e^{-[x(i, j)-x(i-1, j)]^{2} / \gamma} \\
-\gamma e^{-[x(i, j)-x(i+1, j)]^{2} / \gamma}
\end{gathered}
$$

In the DAMRF model, the non-convex functions with many local andglobal minimum function, is solved by a setting parameter Kappa. The design of kappa is initially a very small value. An another parameter gamma and target gamma makes the non-convex function to a convex function, as given by Eq. (09) and Eq. (10). And is written as

$$
\begin{aligned}
=\frac{\partial}{\partial x}\left(\sum_{i} \sum_{j} 4 \gamma\right. & -\gamma e^{-[x(i, j)-x(i, j-1)]^{2} / \gamma} \\
& -\gamma e^{-[x(i, j)-x(i, j+1)]^{2} / \gamma} \\
& -\gamma e^{-[x(i, j)-x(i-1, j)]^{2} / \gamma} \\
& \left.-\gamma e^{-[x(i, j)-x(i+1, j)]^{2} / \gamma}\right)
\end{aligned}
$$

It is known that the blur kernel is a function of $\sigma$ which in turn is a function of depth $\mathrm{d}$. So, we will use these relations and find the gradients required in each case. Image deblurring algorithm using non-convex prior is given in algorithm 1 .

\section{METHODOLOGY / APPROACH}

We get the initial course estimate of the blur-map by JNB [4] techniques by reducing feature map ' $\mathrm{f}$ ' values and is given by

$$
f=\frac{a}{1+\exp (b \sigma+c)}+d \text { relations }
$$

Where $\sigma$ is given by

$$
\sigma=\frac{\log _{e}\left(\frac{a}{f-d}-1\right)-c}{b}
$$

where a, b, c and d are constants with values $39.49,4.535,-3.538$, and 18.53 respectively. Using JNB for ramp blur we get a good blur estimate in the range of (0.4 to 0.95) for both increasing and decreasing ramp as shown in Fig 1 (a) and Fig.4( b). So, we assume it to be almost focused image (near focus) in this range since the blur is very small. Now, we take two blur images one with increasing and other with decreasing ramp in the range of $(0.4$ to 1.5$)$. So, one image has less blur on the left side and more blur on the right side and vice versa for the other image. Since, we get good blur estimate in 0.4 to 1.5 range from image and deblur them separately using Algorithm 1, and Algorithm 2 respectively and get de-blurred images. Now, we have the blur map of the observed image which we get from Algorithm 1 and 2 respectively. Finally, we estimate blur using sigma estimate model as shown in Fig 1 


\section{Estimation of focused image}

The formation of space variantly blurred images $y_{p}(i, j)$ is given by

$$
y_{p}(i, j)=\sum_{k} \sum_{l} x(k, l) h_{p}(i, j ; k, l)+\eta
$$

Here the $x(k, 1)$ is the focused image, $\eta$ is the AWGN given by [6], $h_{p}(i, j ; k, l)$ is the point spread function (PSF) of the lens used setup modeled as a 2D Gaussian function given by

$$
h_{p}(i, j ; k, l)=\frac{1}{2 \pi \sigma_{p}^{2}(i, j)} \exp \left(-\frac{(i-k)^{2}+(j-l)^{2}}{2 \sigma_{p}^{2}(i, j)}\right)^{(17)}
$$

where the standard deviation of the Gaussian function $\sigma_{p}(i, j)$ is the space varying blur parameter at $(i, j)$ in the observation [14]. The Gaussian PSF $h_{\mathrm{p}}(i, j)$ spans the rectangle defined by $(i-3 \sigma(i, j), j-$ $3 \sigma(i, j))$ to $(i+3 \sigma(i, j), j+3 \sigma(i, j))$ centered at $(i, j)$. So, the image blurring can also be modeled as

$$
y_{p}(i, j)=\sum_{k} \sum_{l} x(k, l) h_{k_{p}}(i, j ; k, l)+\eta
$$

The formation of a space variant blurred image can be modeled as

$$
y=X h_{k_{p}}+\eta
$$

where $\mathrm{X}$ is the focused image, $\mathrm{h}$ is the blur kernel and $\eta$ is the additive white zero mean gaussian noise [6]. The results of both equations must be identical. The problem of structure estimation can be formulated as the minimization of the energy function given by

$$
e=\frac{1}{2}\left\|y-X h_{k_{p}}\right\|_{2}^{2}
$$

For solving this ill-posed problem, we need to add regularizer or prior term to smooth-en the outliers and to make it well-posed problem.

$$
\text { So, } E=\frac{1}{2}\left\|y-X h_{k_{p}}\right\|_{2}^{2}+\lambda R(d)
$$

$R(d)$ is a convex function in the range $(\sqrt{-\gamma / 2}, \sqrt{\gamma / 2})$.

$$
R(d)=\gamma-\gamma e^{-\eta^{2} / \gamma}
$$

\section{RESULTS}

For deblurring, the degraded image is synthetically generated by space-variant blur-map. The point by point convolution operation is used as given in the Eq. 17.

The blur-map estimation obtained by our proposed framework using GNC-DAMRF prior for text image is shown in Fig 3. (a) Fig 3. (b) shows the estimated latent text image. The ground truth image of the text used for deblurring using optimization based technique is given in Fig. 1. The generated image by convolving ramp shaped blur-map is shown in Fig 2.

Fig. 4 (a) shows the space-variant blur-map with sigma varying from 0.4 to 1.5 . The images are blurred with space-variant blur using this sine-sigma blur-map. Theestimated sine blur-map using our algorithm is shown in Fig 4 (b) respectively.

Fig. 5 (a) and (c) shows space variantly blurred image. (b) and (d) shows deblurred latent image using our proposed non-convex Model.

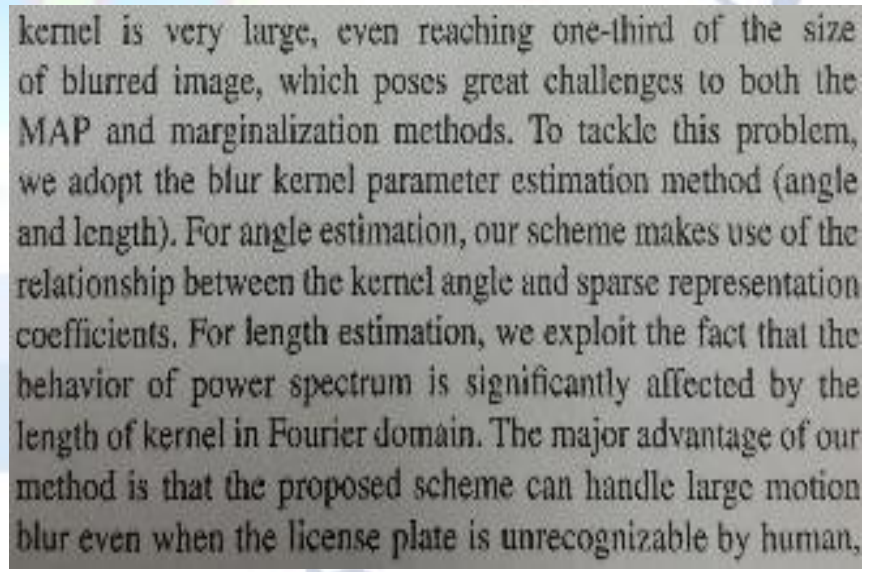

Fig 1. Ground truth Image

The synthetically generated space-variant blurred image using ramp-based sigma-map is shown in fig 4. The estimated Space-variant blur-map used to generate blurred image is hown in fig 5 .

\section{And is given by}




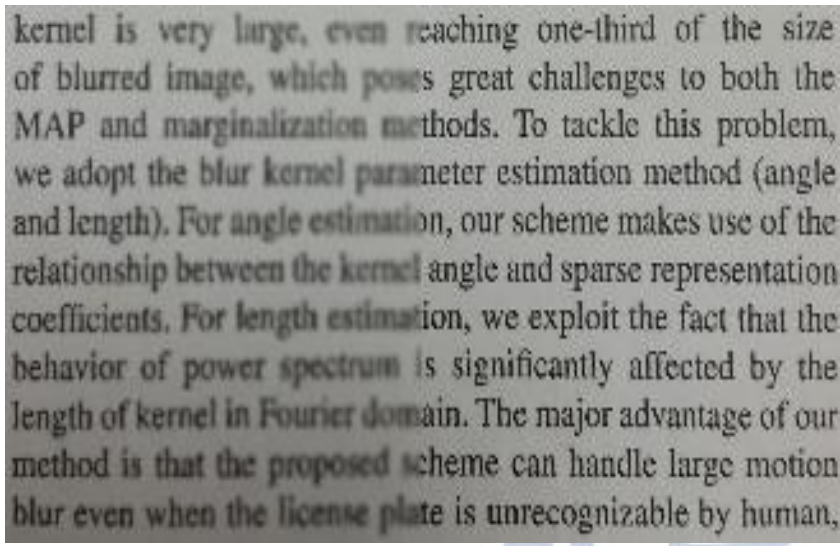

Fig 2. Space-variant blurred mage

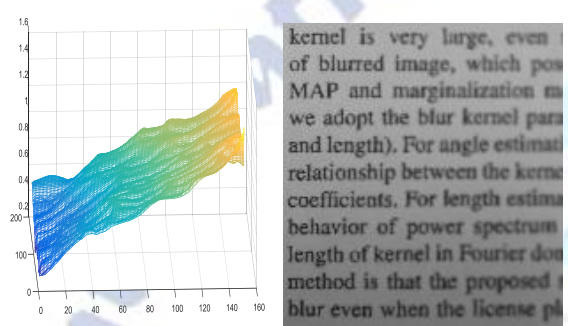

Fig. 3 (a) Estimated blur map of text image (b) Deblurred image
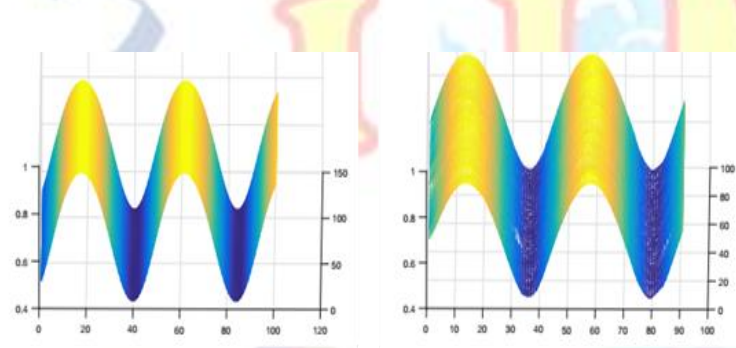

(b)

Fig 4: Blur-map with sigma (0.4 to 1.5 ) (a)Ground truth (b) Estimated blur-ma respectively

aser standies are menelled. In part ral-purpose interactive techniqua ncreasing number of applicatio is mamber of user commmanitics. it tama whether Cand two what exts mingues is influcroced by differes er peopulations. Thesse differences coomitive and persomality trait ce, curkosity. visaal memory. n al someses.

(a)

unor studion aro nocdod. In par ral-purpono intoraotive techntal norcaning number of npplicati. : number of uner comamumition. i tand whothor (and to vethat oxte

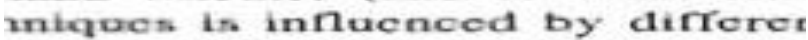
or popalationn. Thase aifroronoca copmituvo and pormommitiy trait co. carionity. Vinual momory. n al apood.

(b) 1 cocdware wass condwactied utilizang n stcm (SASS). This overall test

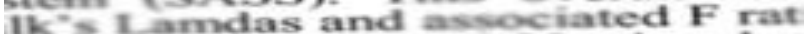
peadent variables involve the a thathavioral val cermined that the bethavioral val mificant impact upom the sil riables are leadersthip style CF 902) and the interanction effect $s$ cass of control CF ratio 2,52 ; P-vals

aving determined that there anificamed in this model, univarial ene them gencrated fore each deper (c)

coduro wan conductod utimeng

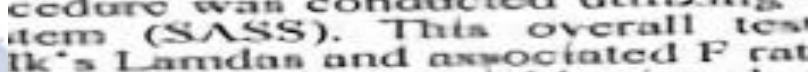

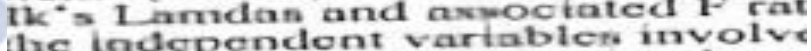
co trat tho beltaviaral va

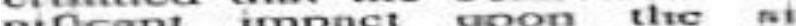
nincant impact cyom tro ni inbica. aro londorsfip ntylo (F 102 ) and tho interaction olToct un of control (1 ratio 2.52 : P-val

iving dotorminod that thoro trefecanco in thin modol. cumivaria ro duen iterieratod for eneli depor

(d)

Fig. 5 (a) and (c) Space variantly blurred image. (b) and (d)

Deblurred latent image using our proposed non-convex Model

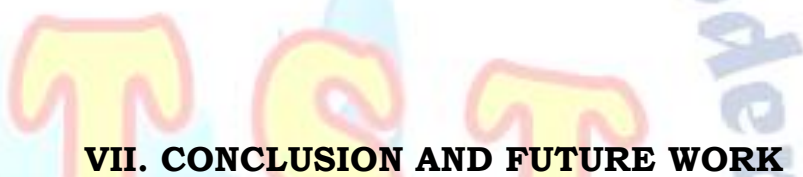

We have proposed a novel work based on sparsity optimization framework capable of estimating blur-map of different shape similar to increasing blur-sigma, decreasing blur-sigma, sine sigma and shifted sine sigma for Broadz Calf image data and DSLR captured text image. The results obtained both in-terms of quality and quantity is better than the few state-of-the art work given the literature. Deblurring of images using gradient descent optimization algorithm with Gaussian Image understanding: Semantic Segmentation of Graphics and Text using Faster-RCNN Markov random field prior is proposed. Depth-map estimation of images using JNB sigma-map features generated. Future work is extended for large depth map estimation of real images. Presently the proposed work is a very restrictive model since the blur-map estimation works only for $0<\sigma \leq 2$.

\section{REFERENCES}

[1] A. N. Rajagopalan and S. Chaudhuri, "An MRF model based approach to simultaneous recovery of depth and restoration from defocused images," Pattern Analysis and Machine Intelligence, IEEE Transactions on, vol. 21, no. 7, pp. 577-589, 1999

[2] L. Xu, S. Zheng, and J. Jia, "Unnatural 10 sparse representation for natural image deblurring," in Proc. CVPR, 2013, pp. 1107-1114.

[3] J. Pan, D. Sun, M.-H. Yang, and H. Pfister, "Blind image 
deblurring using dark channel prior," in Proc. CVPR, 2016. [4] J. Shi, L. Xu, and J. Jia, "Just noticeable defocus blur detection and estimation," in Computer Vision and Pattern Recognition (CVPR), 2015 IEEE Conference on, pp. 657-665, IEEE, 2015.

[5] Anat Levin, Yair Weiss, Fredo Durand, and William T Freeman. Efficient marginal likelihood optimization in blind deconvolution.In IEEE Conference on Computer Vision and Pattern Recognition, 2011.

[6] Robert Fergus, Barun Singh, Aaron Hertzmann, Sam T. Roweis, and William T. Freeman. Removing camera shake from a single photograph. ACM Transactions on Graphics, 25(3):787-794, 2006.

[7] Sunghyun Cho and Seungyong Lee.Fast motion deblurring. ACM Transactions on Graphics, 28(5):145, 2009.

[8] Qi Shan, JiayaJia, and AseemAgarwala. High-quality motion deblurring from a single image. ACM Transactions on

Graphics, 27(3):73, 2008.

[9] Li Xu and JiayaJia.Two-phase kernel estimation for robust motion deblurring.In European Conference on Computer Vision, 2010.

[10] Li Xu, Shicheng Zheng, and JiayaJia.Unnatural 10 sparse representation for natural image deblurring.In IEEE Conference on Comp. Vision and Pattern Recognition, 2013.

[11] Latha, H. N., Palachandra, M. V., \& Rao, M. Real Time Implementation and Performance Evaluation of WCDMA System over AWGN Channel on TMS320C6713DSK.Procedia Technology, 4, 82-86. 2012

[12] S. Z. Li, Markov random field modeling in computer vision. Springer Science \& Business Media, 2012.

[13] A. Chakrabarti, T. Zickler, and W. T. Freeman, "Analyzing spatially-varying blur," inCVPR,IEEE Conference on, pp. 2512-2519, IEEE, 2010.

[14] Latha H N, KrantiK.P, and Sahay R R, "Simultaneous blur map estimation and deblurring of a single space-variantly defocused image." $201727^{\text {th }}$ National Conference on Communications (NCC).IEEE, 2017.

[15] Lerenhan Li, Jinshan Pan, Wei-Sheng Lai, ChangxinGao, Nong Sang, and Ming-Hsuan Yang. Learning a discriminative prior for blind image deblurring.In IEEE Conference on Computer Vision and Pattern Recognition, 2018.

[16] Latha H N, Lakshmi M V and Ramachandran s " Design Of Context Based Adaptive Variable Length Coding And Deblocking Filter for H. 264" Elsevier, Science Direct, PP 671676, 2012.

[17] Sheeba S and Latha H N, "Design of Enhanced Visual Saliency Detection model in H. 264/AVC" International Journal for Science and Engineering Technology Research Vol 6, issue 8, June 2014

[18] Y.-W. Tai and M. S. Brown, "Single image defocus map estimation using local contrast prior," in Image Processing (ICIP), 2009

[19] Latha H N and H N Poornima 'Estimation of Blur and Depth Map of a Defocused Image by Sparsity using Gauss Markov Random Field Convex-Prior' International Journal for Science and Research, Vol 08, Issue 11, pp 728-733, Nov2019

[20] X. Zhu, S. Cohen, S. Schiller, and P. Milanfar, "Estimating spatially varying defocus blur from a single image," Image Processing, IEEE Transactions on, vol. 22, no. 12, pp. 4879-4891, 2013.

[21] S. Zhuo and T. Sim, "Defocus map estimation from a single image," Pattern Recognition, vol. 44, no. 9, pp. 1852-1858, 2011.

[22] Sheeba S and Latha H N, " Design \& Implementation of Saliency Detection Model in H. 264 Standard” IJSR, pp14-2020, 2014

[23] A. Chakrabarti and T. Zickler, "Depth and deblurring from a spectrally-varying depth-of-field," in Computer Vision-ECCV 2012, pp. 648-661, Springer, 2012.

[24] S. Dai and Y. Wu, "Removing partial blur in a single image," in Computer Vision and Pattern Recognition, 2009. CVPR 2009. IEEE Conference on, pp. 2544-2551, 2009.

[25] N. Joshi, R. Szeliski, and D. J. Kriegman, "Psf estimation using sharp edge prediction," in Computer Vision and Pattern Recognition, 2008. CVPR 2008.IEEE Conference on, pp. 1-8, IEEE, 2008.

[26] Latha, H. N., and BharathiLokesh. "Denoising and Deblurring by Gauss Markov Random Field: An Alternating Minimization Convex Prior."IJSR : 2319-7064, Nov 2019. 8 (11),

6

[27] HS Meharwade, HN Latha, Design of high speed face detection and tracking by skin segmentation using matlab International Journal of Engineering Science and Research (IJESR), 1-6, 2014

[28] HN Latha, S Rudresh, D Sampreeth, SM Otageri, " Image understanding: Semantic Segmentation of Graphics and Text using Faster-RCNN "International Conference on Networking, Embedded and Wireless Systems(ICNEWS). 14-17, DEC 2018. [29] Latha H N, Rajiv R. Sahay and H N Poornima Simultaneous Denoising and Deblurring by Non-Convex

Regularization: Alternating Minimization Framework" in IEEE conference ICCSP 2020, 401-406, July 2020.

[30] Latha H N, and Rajiv R. Sahay “Joint Estimation of Depth Map and Focus Image in SFF: An Optimization Framework by Sparsity Approach" in IEEE conference ICCSP 2020, 401-406, July 2020.

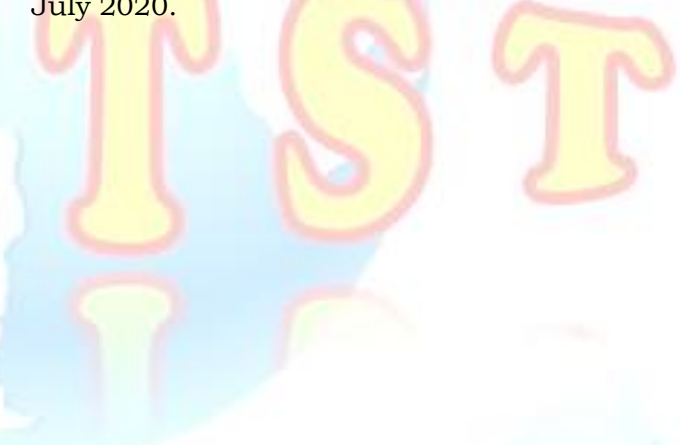

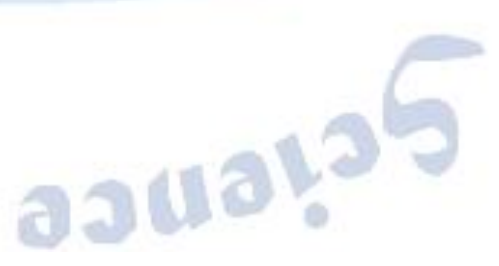

